\title{
GLOBAL JOURNAL OF
}

\section{Community Psychology Practice}

\section{PROMOTING COMMUNITY PRACTICE FOR SOCIAL BENEFIT}

\section{The Living Room, a Community Crisis Respite Program: Offering People in Crisis an Alternative to Emergency Departments}

\author{
Michelle Heyland, MSN, APN, PMHNP-BC \\ Courtney Emery, MA, LCPC \\ Mona Shattell, PhD, RN
}

\section{Author Notes:}

Michelle Heyland, MSN, APN, PMHNP-BC, is faculty at Rush University College of Nursing in Chicago, IL, USA, and a Psychiatric \& Mental Health Nurse Practitioner at Turning Point Behavioral Health Care Center in Skokie, IL, USA.

michelle_heyland@rush.edu

Courtney L. Emery, MA, LCPC, is the Assistant Program Director for The Living Room and Community Support Services at Turning Point Behavioral Health Care Center in Skokie, IL, USA.

Mona M. Shattell, PhD, RN, is the Associate Dean for Research and Faculty Development at DePaul University's College of Science and Health and an Associate Professor at DePaul University's School of Nursing in Chicago, IL, USA.

Keywords: crisis, recovery, peer support, hospital diversion, emergency department diversion, emergency department alternative

Recommended citation: Heyland, M., Emery, C., \& Shattell, M. (2013). The Living Room, a Community Crisis Respite Program: Offering People in Crisis an Alternative to Emergency Departments. Global Journal of Community Psychology Practice, 4(3), 1-8. Retrieved Day/Month/Year, from (http://www.gjcpp.org/). 


\title{
The Living Room, a Community Crisis Respite Program: Offering People in Crisis an Alternative to Emergency Departments
}

\begin{abstract}
Objective: To describe The Living Room, a community crisis respite center that offers individuals in crisis an alternative to obtaining services in an emergency department (ED).

Methods: This article describes the problems individuals in a mental health crisis may encounter in traditional EDs and explains how The Living Room addresses these problems. The Living Room's development, setting, staffing and procedures are described in order to promote increased use of this type of program.

Results: In its first year of operation, The Living Room hosted 228 visits by 87 distinct individuals (termed "guests"). Guests were deflected from EDs on 213 of those visits - a 93\% deflection rate. These deflections represent a savings of approximately $\$ 550,000$ to the State of Illinois since guests of The Living Room are overwhelmingly individuals with Medicaid or no insurance of any kind. On $84 \%(\mathrm{n}=192)$ of the occurrences in which guests were deflected from EDs, they alleviated their crises sufficiently to decide to leave The Living Room and return to the community. These guests reported an average decrease of 2.13 points on the Subjective Units of Distress Scale.
\end{abstract}

Conclusions: Community crisis respite centers such as The Living Room represent an important alternative to EDs by remedying many criticisms of traditional EDs made by individuals in crisis. Outcomes from The Living Room's first year of operation suggest that community crisis respite centers are cost-effective, effective in helping many individuals alleviate crises, and have the potential to decrease the use of EDs for mental health crisis.

Keywords: crisis, recovery, peer support, hospital diversion, emergency department diversion, emergency department alternative

\section{Introduction}

People in crisis, whether or not they have a diagnosed mental illness, are often faced with a dilemma at the height of their distress: do they stay at home and hope the intense emotions dissipate or do they go to a hospital's emergency department (ED)? Unfortunately, due to a lack of service alternatives in the community, many clients decide to go to local EDs. Once in the ED, individuals with mental illness are often met with frustration, disregard or minimization of symptoms (Shattell, 2011). Furthermore, their symptoms may be easily exacerbated because of the nature of EDs: crowded, loud, and containing multiple and unsettling stimuli. Clarke, Dusome, and Hughes (2007) examined the experiences of a general hospital ED visit by individuals with mental illness and identified several issues. The study reported participants saying they were not sick enough to receive priority triage, they experienced a lack of privacy in the triage area that exacerbated distress, and that the ED staff lacked the expertise to address psychiatric conditions. They also experienced shame or guilt for presenting to the ED and for having a mental illness. Finally these participants reported that their families wanted to be more involved and supported during the time of crisis (Clarke et al., 2007).

Studies identified what individuals find helpful during mental health crises. For example, Clarke et al., (2007) reported that clients in crisis desire a safe place that is open around-the-clock and staffed with strong advocates. Similarly, Agar-Jacomb and Read (2009) concluded that clients in crisis sought a safe place with people who they can talk to and who can understand what they were going through. These clients also preferred access to trained professionals, autonomy, physical freedom, and respect (Agar-Jacomb \& Read, 2009). Furthermore, clients described that in a time of crisis they need, "somewhere safe to go away from [their] normal life," and, "someone to talk to but not in hospital" (Lyons et al., 2009, p. 429). Without such elements, a client's psychiatric crisis may escalate. 
There is increasing recognition among mental healthcare professionals that improvements need to be made to current crisis intervention services. Specifically, "Alternatives to admission need to be seen as viable options that address concerns of families and individuals with mental health needs rather than be perceived as a 'brush off' of their concerns" (Clarke et al., 2007, p. 130).

The purpose of this paper is to describe one alternative, a community crisis respite program called The Living Room. We will report the development of The Living Room; the space, location, and funding; staffing; procedures; and preliminary outcomes related to treating emotional distress and diverting persons in crisis from EDs.

\section{A Community Crisis Respite Program: The Living Room}

\section{Background}

Communities have begun to implement programs that provide appropriate care to people in crisis and are a cost effective alternative to EDs. One such initiative is a community crisis respite program called The Living Room. Although community crisis respite programs of this type are relatively rare in the US they are increasingly recognized as an effective way to reduce psychiatric ED visits while improving outcomes for people in crisis. While a focus of The Living Room is to help those in crisis avoid using the ED, the service philosophy also embraces the Recovery Model. The concept of recovery "Involves the development of new meaning and purpose in one's life as one grows beyond the catastrophic effects of mental illness" (Anthony, 1993). The Living Room reflects many of the Recovery Model's concepts, including autonomy, respect, hope, empowerment, and social inclusion.

The Living Room is named after the familiar home space and offers a safe destination for those in crisis. It is located in Skokie, IL, a Chicago suburb, and is easily accessible via public transportation. The space is in the same building as a well-established and financially stable community mental health center; as a result, the location is readily identifiable to the public. The hours of the crisis respite center depend largely on need. For example, The Living Room selected its hours of operation (Wednesday, Thursday, and Sunday from 38PM) based on the high volume of calls received on the community mental health center's Emergency Services hotline. The time also aligned with those that area ED personnel reported as high volume periods of psychiatric visits.

Clients who utilize The Living Room are referred to as "guests" in order to communicate the intent of The Living Room to be a non-clinical environment with an inviting atmosphere. The use of the term is also meant to imply that services are person-centered. Appropriate guests of The Living Room are individuals 18 years and older who are experiencing acute crises sufficiently severe that they are at risk for ED visits. Examples include individuals who are experiencing suicidal or homicidal thoughts, panic attacks, situational crises, severe depressive symptoms, and psychotic symptoms. The Living Room is available regardless of where the person lives, their type of insurance coverage, and the exact nature of their crisis. Individuals need not have a diagnosed mental illness to utilize The Living Room.

The Living Room can comfortably accommodate up to six guests at any given time. The capacity is set at six to ensure that guests do not have to wait to meet with a mental health professional. Minimizing wait time is important considering long wait times are among the biggest sources of dissatisfaction for people in crisis who visit EDs (Clarke et al., 2007). To date, no guests have had to be turned away because The Living Room exceeded maximum capacity.

The Living Room was originally funded by a special projects grant from the State of Illinois' Division of Mental Health (DMH); so unlike services delivered in EDs, there are never any out-of-pocket costs for guests of The Living Room. In summer 2012 this special projects grant was renewed for a second year, but even with these monies the center staff realized that to ensure adequate long-term funding they needed to have additional revenue. To that end, for a subset of clients, The Living Room now bills Medicaid or Illinois' DMH for crisis intervention services. Specifically, Medicaid provides reimbursement for Medicaid-eligible clients of the community mental health center in which The Living Room resides. Illinois' DMH provides reimbursement for clients of the agency with no insurance of any kind.

\section{Setting}

An outpatient mental health agency was the ideal setting for the establishment of The Living Room due to ease of access to professional staff and widespread familiarity with the agency. The environment of the actual space is warm and welcoming with carpeted floors, paintings (art) on the walls, comfortable furniture, and soft lighting. The environment was designed so that guests are likely to feel safe and not overwhelmed by excessive stimuli (e.g., television, radio or excessive number of people). The layout of the space ensures maximum privacy and includes a separate space for rest and relaxation. A psychiatric registered nurse who conducts client intakes uses a space away from the main meeting area. The nursing area utilizes a small private office that is carpeted and boasts only a bookshelf, desk, and chairs across the hall 
from The Living Room. The nursing area allows for privacy and does not appear like a stark clinical exam room so as to not increase guest anxiety.

\section{Staffing and Roles}

The Living Room utilizes one counselor, one psychiatric registered nurse, and three peer counselors for staffing on a regular basis, all of whom have extensive experience working with persons in crisis. The counselor conducts an initial triage, determines whether the guest is safe to utilize The Living Room, and completes a petition in the event that a guest is at high risk of hurting him- or herself or someone else and thus requires an emergency psychiatric hospitalization. The psychiatric registered nurse ensures that guests are medically stable with a basic set of vital signs and other health screening measures (e.g., glucose testing if symptomatic of hypoglycemia). Recognizing that medical concerns can also be precursors to crises, the psychiatric registered nurse provides health and medication education to guests and also assists in therapeutic interventions.

An integral part of The Living Room's team is the peer counselors. Peer counselors are individuals who have a mental illness but have achieved sufficient recovery to function as supports to those in crisis. Prior to their employment at The Living Room, the peer counselors all had experience co-facilitating National Alliance on Mental Illness (NAMI) support groups or they had equivalent training. After they were hired by The Living Room, the peer counselors received extensive training in crisis assessment, de-escalation, active listening, and operations of The Living Room. The peer counselors also attended an intensive Peer Support Specialist training program sponsored by the Depression and Bipolar Support Alliance.

Peer counselors in The Living Room meet with guests to de-escalate crises and establish achievable short-term goals, develop safety plans, and teach coping skills. Their ongoing professional development is facilitated through weekly individual clinical supervision with The Living Room's program director (a counselor).

Individual clinical supervision provides an opportunity to learn new clinical skills, process the application of clinical skills to the needs of guests, and understand their own reactions to guests and other experiences within The Living Room. Peer counselors have been shown to greatly improve outcomes for those in crisis because they meet the need to talk to someone who can truly understand the person in crisis (Optum Health, 2011; Agar-Jacomb \& Read, 2009).

\section{Procedures}

When a guest initially arrives in The Living Room, the counselor triages the individual to obtain basic demographics, determine the nature of the guest's crisis, and assess his/her level of risk (e.g., lethality of suicidal thoughts, plans, and intent). The triage form includes the Subjective Units of Distress Scale (SUDS), a self-reported measure of an individual's level of distress based on descriptive definitions of what each number (zero through ten) represents in terms of distress level. This measure was selected because of its ease of use and documented reliability and validity (Benjamin, O’Neil, Crowley, \& Beidas, 2010; Furukawa, Kessler, Slade, \& Andrews, 2003; Kaplan \& Smith, 1995; Kessler, Andrews, Colpe, Hiripi, Mroczek, Normand, Walters, \& Zaslavsky, 2002; Tanner, 2012). Each guest is asked to rate his/her distress level upon arrival. This initial distress level helps the staff and guest determine the effectiveness of The Living Room (as it is compared to an exit distress level obtained at departure). The counselor also decides if the guest is appropriate for The Living Room. For example, the guest is deemed not appropriate if under the influence of drugs or alcohol. This assessment is made through guest self-report, observation of clinical signs and symptoms, and findings from the nursing assessment (e.g., dilated pupils and accelerated heart rate). In this case the individual is given a list of resources to utilize once they leave The Living Room. Examples of these resources include local Alcoholics Anonymous/Narcotics Anonymous meetings, substance abuse treatment providers, and crisis hotlines.

Throughout the visit, assessment of the guest's safety is ongoing. If at any point the counselor determines the guest poses an immediate and emergent safety threat to self or others, a petition for involuntary psychiatric hospitalization is completed and an ambulance is called to safely transport the individual to a local ED for further evaluation. If the guest refuses to leave in an ambulance and a petition has been completed, the police are called. If the guest leaves before the ambulance arrives, the police are also called to make every effort to locate the guest to ensure his/her safety. In the vast majority of cases, a petition is avoided because the guest stabilizes over the course of his/her visit in The Living Room. Guests are never held involuntarily in The Living Room; therefore, chemical or mechanical restraints are never used.

After meeting with the counselor, the guest meets with the psychiatric registered nurse for a basic health assessment because physical and emotional needs are often intertwined. The psychiatric registered nurse initially obtains the guest's height and weight and vital signs (blood pressure, heart rate, respiratory rate, and temperature). If the nurse determines that the vital signs are dangerously abnormal or the client is symptomatic of a medical emergency (e.g., chest pain and shortness of breath), the nurse calls 911 and the client is 
transported to the nearest ED for medical evaluation. The nurse also answers any questions the guest may have regarding health concerns or medications. The nurse assists in de-escalation, empathic listening, support, and ensures that the whole person (mental and physical) is addressed. Respecting autonomy, guests have the right to refuse a visit with the psychiatric registered nurse. However, under certain circumstances the guest is required to meet with the psychiatric nurse. For example, a guest must meet with the nurse if he/she is exhibiting emergent symptoms such as chest pain or a sudden and severe headache or if a guest has a sudden change in physical or mental status such as disorientation, confusion or difficulty speaking.

Following the meeting with the psychiatric registered nurse, the guest is paired with a peer counselor. The pairing process ensures each peer counselor has sufficient guest experiences and that the pairings are appropriate (e.g., a male guest with a known history of predatory-type behaviors would not be paired with a female peer counselor). The peer counselor and the guest discuss in-depth the nature of the crisis and coping skills the guest might use to decrease distress. The use of coping skills is considered one of the main pillars of the Recovery Model, encouraging the development of self-empowerment (Anthony, 1993). Two peer counselors may also work together with certain guests who are more complex or require greater immediate support. Using knowledge gained through their own personal experiences with mental illness, peer counselors are uniquely positioned to offer support and empathy and to advocate for guests.

Guests can leave The Living Room at any time, exercising their right to autonomy (sans a completed petition for the guest). When a guest determines that he/she is ready to leave The Living Room, the counselor meets with him/her for a final assessment to ensure the guest is indeed safe to leave and to determine if the guest's distress level has decreased. Most guests come to The Living Room with the goal of avoiding an ED if possible, and they are able to make use of the services available to achieve this outcome.

\section{Outcomes}

Initial outcomes of The Living Room are positive. Guests have been diverted from EDs, the State of Illinois has realized a cost savings, and guests have received care for their emotional distress as evidenced by the average decrease in SUDS scores. In its first year of operation, The Living Room hosted 87 distinct guests and a total of 228 visits. Guests routinely come to The Living Room with the goal of avoiding an ED if at all possible. The Living Room is widely promoted to offer people in crisis an alternative to area EDs. Therefore, it is possible that if The Living Room did not exist, these guests would have elected to go to an ED. Assuming this, guests were successfully deflected from EDs on 213 of those visits - a 93\% deflection rate. A deflection is defined by The Living Room as a visit that does not result in a trip to the ED and instead with the guest returning to the community when sufficient relief from distress has been achieved.

The ED deflections represent a savings of approximately $\$ 550,000$ to the State of Illinois. Guests of The Living Room are overwhelmingly individuals with Medicaid or no insurance of any kind. Each ED deflection represents a savings of approximately $\$ 2631$, the difference between the average cost of a visit to a local ED for a psychiatric reason (\$2900 on the low end of a six hospital range) and the average cost of a visit to The Living Room (\$269). The average cost of an ED visit for a psychiatric reason is based on data provided by the Illinois Department of Public Health (Illinois Department of Public Health, Office of Policy, Planning and Statistics, 2010). The average cost of a visit to The Living Room is calculated to include staff time, materials, and a small share of the operating costs of The Living Room's parent agency.

Most guests of The Living Room experience less distress by the end of their visit. On $84 \%(n=192)$ of the visits in which guests were successfully deflected from EDs, guests alleviated their crises sufficiently to leave The Living Room. These guests reported an average decrease of 2.13 points on the SUDS. On the remaining $9 \%(n=21)$ of visits, guests were sufficiently stable to avoid ED referrals, but did not fully resolve their crises within The Living Room. In most cases staff in The Living Room linked these guests to longer-term treatment.

Anecdotal reports by The Living Room guests frequently express relief and amazement that such an alternative exists, as these quotes illustrate. "I am so glad I came here and that you are real and can relate to me. I'm sick of going to the ER." "At the ER, it's very frantic, there are no choices. Here I was able to talk and calm down. I might have even ended up in the hospital for a week for saying I was suicidal, but here I could tell you and you gave me a chance to calm down without sending me to the hospital." These statements describe the value of ED alternatives such as The Living Room.

Although preliminary outcomes of The Living Room are positive, research is needed to more systematically understand and evaluate this alternative treatment environment. A study is currently underway that aims to describe the environment from the perspective of persons who spend time there - guests and staff. 


\section{Global Journal of Community Psychology Practice}

\section{Conclusions}

Emergency departments often provide inadequate services for people in crisis. Persons in emotional distress frequently report negative experiences in such chaotic environments. Conversely, ED staff may become frustrated with over-crowding and perceived "frequent fliers." Individuals with mental illness can be stereotyped as disruptive or dangerous, which may decrease the quality of care that they receive. Such clients are acutely aware of how they are perceived in EDs, and while they understand that EDs are unlikely to meet their needs they see no other options.

Community crisis respite centers therefore represent an important alternative to EDs. Community crisis respite centers such as the one described here - The Living Room - remedy the criticisms of EDs that people in crisis often voice by providing care that is immediate, client-centered, and recovery-oriented. Furthermore, people in crisis who visit community crisis respite centers have better outcomes than those who visit traditional emergency departments (Adams \& ElMallakh, 2009; Greenfield, Stoneking, Humphreys, Sundby, \& Bond, 2008). Outcomes from The Living Room's first year of operation suggest that community crisis respite centers are more cost-effective than traditional EDs and that such centers are effective in helping many individuals alleviate crises without the use of EDs.

Unfortunately, resources in the US to open such centers are not readily available. Early outcomes in The Living Room should lend support for funding and allocation of resources to open additional similar programs. These outcomes suggest that such programs offer individuals in crisis more suitable interventions while saving money. At a time when overcrowding in EDs is recognized as a national problem, this has never been more important.

\section{References}

Adams, C.L., \& El-Mallakh, R.S. (2009). Patient outcome after treatment in a community-based crisis stabilization unit. Journal of Behavioral Health Services \& Research, 36(3), 396-399.

Agar-Jacomb, K. \& Read, J. (2009). Mental health crisis services: What do service users need when in crisis? Journal of Mental Health, 18(2), 99-110. doi: 10.1080/09638230701879227

Anthony, W.A. (1993). Recovery from mental illness: The guiding vision of the mental health service system in the 1990s. Psychosocial Rehabilitation Journal, 16(4), 11-23.
Benjamin, C.L., O’Neil, K.A., Crowley, S.A., \& Beidas, R.S. (2010). Patterns and predictors of subjective units of distress in anxious youth. Behavioural and Cognitive Psychotherapy, 38, 497-504. doi: 10.1017/S1352465810000287

Clarke, D.E., Dusome, D., \& Hughes, L. (2007). Emergency department from the mental health client's perspective. International Journal of Mental Health Nursing, 16, 126-131. doi: 10.1111/j.1447-0349.2007.00455.x

Furukawa, T.A., Kessler, R.C., Slade, T., \& Andrews, G. (2003). The performance of the K6 and K10 screening scales for psychological distress in the Australian National Survey of Mental Health and Wellbeing. Psychological Medicine, 33, 357-362.

Greenfield, T.K., Stoneking, B.C., Humphreys, K., Sundby, E., \& Bond, J. (2008). A randomized trial of a mental health consumer-managed alternative to civil commitment for acute psychiatric crisis. American Journal of Community Psychology, 42(1-2), 135-144.

Illinois Department of Public Health, Office of Policy, Planning and Statistics (2010). Average Emergency Department Costs. Springfield, IL.

Kessler, R.C., Andrews, G., Colpe, L.J., Hiripi, E., Mroczek, D.K., Normand, S., Walters, E.E., \& Zaslavsky, A.M. (2002). Short screening scales to monitor population prevalences and trends in nonspecific psychological distress. Psychological Medicine, 32, 959-976.

Lyons, C., Hopley, P., Burton, C.R., \& Horrocks, J. (2009). Mental health crisis and respite services: Service user and carer aspirations. Journal of Psychiatric and Mental Health Nursing, 16, 424433.

Optum Health (2011). Hospital Diversion Services. Golden Valley, MN: Optum Health.

Shattell, M., \& Andes, M. (2011). Treatment of persons with mental illness and substance use disorders in medical emergency departments in the United States. Issues in Mental Health Nursing, 32(2), 140-141.

Tanner, B.A. (2012). Validity of global physical and emotional SUDS. Applied Psychophysiology and Feedback, 37(1), 31-34. doi 10.1007/s10484-0119174-x 


\section{APPENDIX}

\section{Images from The Living Room}

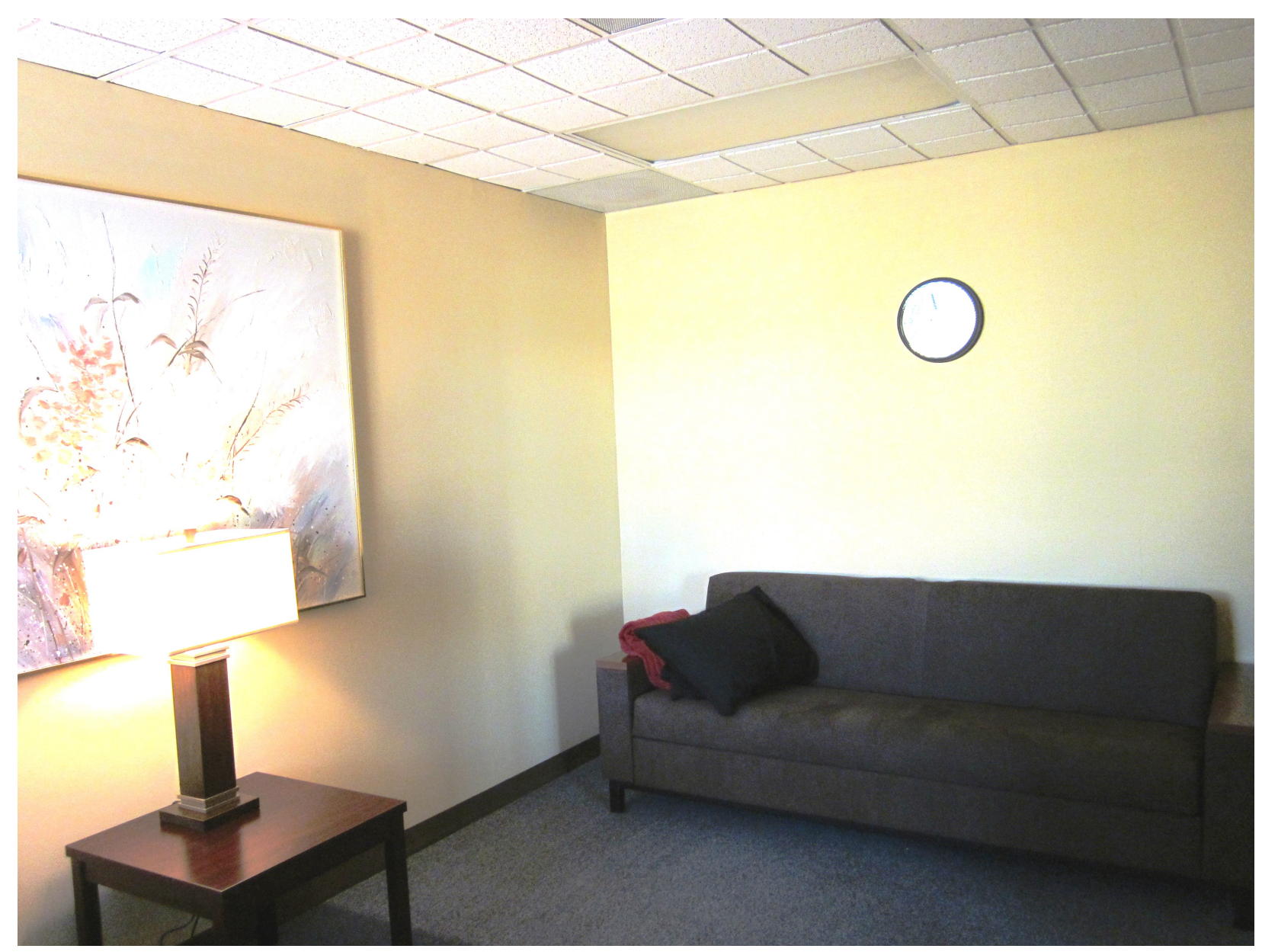

Image 1. R and R Room: The rest and relaxation room where guests can have added privacy or relax alone if necessary. 


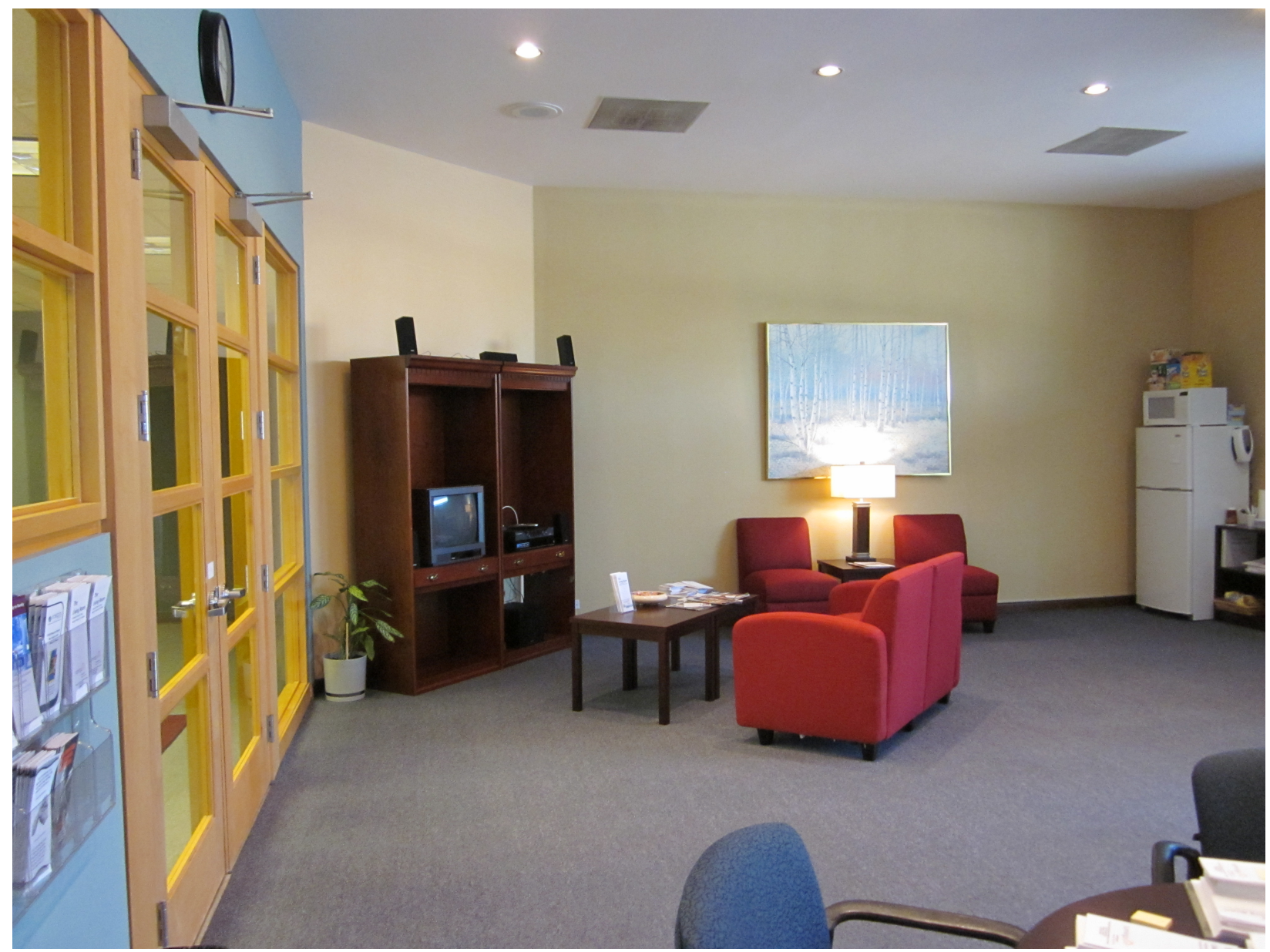

Image 2. One of The Living Room's seating areas. 


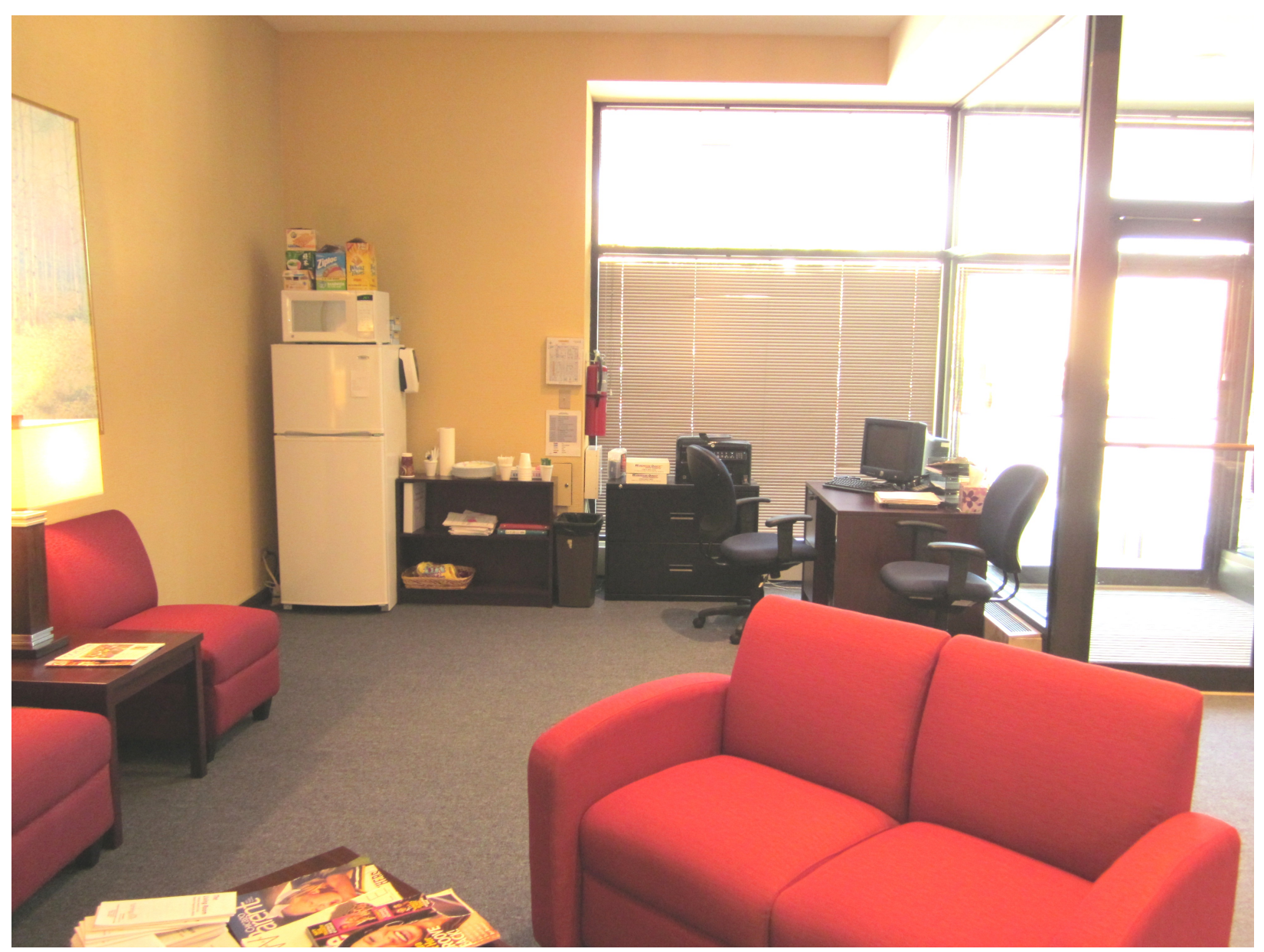

Image 3. The Living Room triage area and a seating area. 


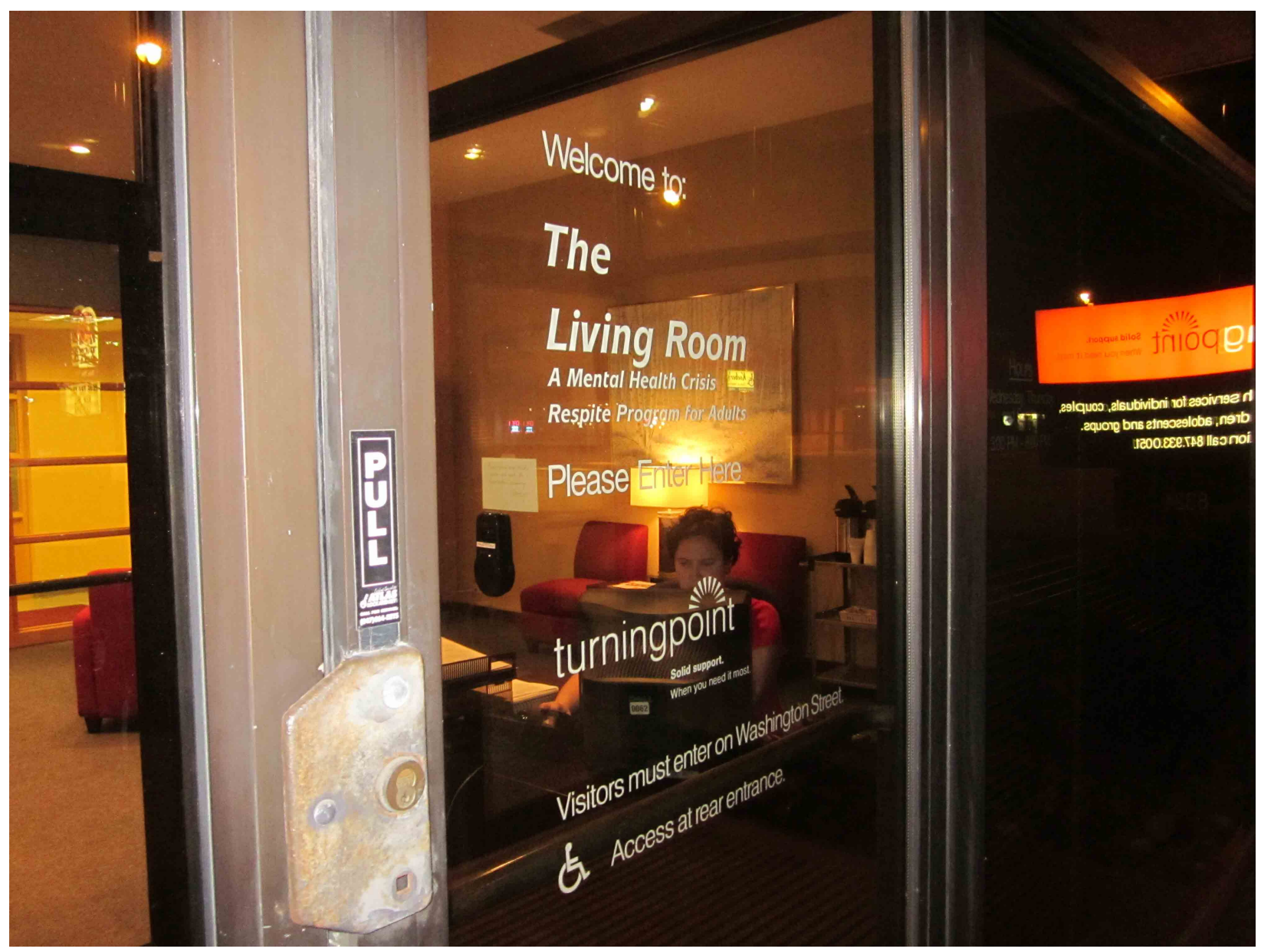

Image 4. Street entrance into The Living Room. 